Portland State University

PDXScholar

$11-1-1989$

\title{
Bethe stopping-power theory for heavy-target atoms
}

P.T. Leung

Portland State University

Follow this and additional works at: https://pdxscholar.library.pdx.edu/phy_fac

Part of the Physics Commons

Let us know how access to this document benefits you.

\section{Citation Details}

Leung, P. T. (1989). Bethe stopping-power theory for heavy-target atoms. Physical Review A, 40(9), 5417-5419.

This Article is brought to you for free and open access. It has been accepted for inclusion in Physics Faculty Publications and Presentations by an authorized administrator of PDXScholar. Please contact us if we can make this document more accessible: pdxscholar@pdx.edu. 


\title{
Bethe stopping-power theory for heavy-target atoms
}

\author{
P. T. Leung \\ Department of Physics, Portland State University, P.O. Box 751, Portland, Oregon 97207-0751
}

(Received 10 April 1989; revised manuscript received 28 June 1989)

\begin{abstract}
The Bethe stopping-power theory is considered in the context of heavy target atoms in which the relativistic effects of the target electrons are partially accounted for within the semirelativistic and independent-particle description. Numerical results show that, for target elements with large atomic numbers, these relativistic effects can become comparable with other corrections such as the shell correction, the Barkas effect, and the Bloch term.
\end{abstract}

The result from the Bethe stopping-power theory ${ }^{1,2}$ for heavy incident particles can usually be expressed as follows:

$-\left(\frac{d E}{d x}\right)=\frac{4 \pi z^{2} e^{4}}{m v^{2}} N Z\left[\ln \left[\frac{2 m \gamma^{2} v^{2}}{I}\right]-\beta^{2}\right]$,

where ze and $Z e$ are, respectively, the charge of the incident particle and the target atom, $v=\beta c$ is the velocity of the incident particle, $N$ the number density and $I$ the mean excitation energy of the target atoms, and $m$ is the mass of the electron. It is well known that while Eq. (1) can be applied to case of high-energy projectiles, it is nevertheless restricted for nonrelativistic target atoms in the sense that the target-atom wave functions must be eigenfunctions of the Schrödinger equation for the manyelectron system. The difficulty in extending Eq. (1) to include the relativistic effects of the target electrons (which are important for heavy target elements) lies of course in its derivation in which the nonrelativistic sum rules have been applied. This includes the Thomas-Reiche-Kuhn (TRK) sum rule for dipole oscillator strength as well as the Bethe sum rule for the generalized oscillator strength of the target atoms. This problem has been recognized as early as the mid 1960 s by Fano, ${ }^{3}$ and not much progress has since been made due to the fact that the generalization of various sum rules to the relativistic domain is not a trivial task as already noted by Fano. ${ }^{3}$

In recent years, however, due to the fundamental and simple nature of the sum-rule approach, there has been much effort in extending it to include relativistic effects $^{4-9}$ and applications to various phenomena such as in the analysis of anomalous $x$-ray scattering data has been made. ${ }^{10}$ The usual methods for such relativistic generalization include the semirelativistic, ${ }^{4-7}$ the projection-operator, ${ }^{8}$ and the field-theoretic approach. ${ }^{9}$ The key point here is to get rid of the negative-energy states of the atomic system and still maintain completeness for the atomic wave functions.

It is the purpose of this Report to apply our previous result for the relativistic correction to the Bethe sum rule $^{6}$ to study the effects on the Bethe stopping-power theory due to the relativistic nature of the electrons for heavy target elements. Since most of the previous results ${ }^{4-8}$ have been derived only for a one-electron system, our application here can only be justified in the limit of the independent-particle, local-potential description for the many-electron atoms, an apparent oversimplified picture which has been found to be reasonably good in a previous analysis of the $\mathrm{x}$-ray scattering data where the TRK sum rule has been applied. ${ }^{10}$ In any case, our result here can at least yield an estimate and a trend for such relativistic effects of the target atoms. We shall restrict ourselves to work only in the case of a nonrelativistic incoming particle in this Report.

For nonrelativistic incident particles, the Bethe theory gives $^{11}$

$$
-\left(\frac{d E}{d x}\right]_{0}=\frac{4 \pi z^{2} e^{4}}{m v^{2}} N Z \int_{Q_{\min }}^{Q_{\max }} \frac{d Q}{Q}|M|^{2},
$$

where $|\boldsymbol{M}|^{2}$ is the sum of the generalized oscillator strength given by

$$
|\boldsymbol{M}|^{2}=\sum_{n}\left|\left\langle n\left|\sum_{i=1}^{Z} e^{i \mathbf{q} \cdot \mathbf{r}_{i}}\right| 0\right\rangle\right|^{2}\left(E_{n}-E_{0}\right) .
$$

For nonrelativistic target atoms, the Bethe sum rule yields ${ }^{1,11}$

$$
|M|^{2}=\frac{\hbar^{2}}{2 m} Z q^{2}
$$

together with the results $\hbar q_{\min }=I / v$ and $\hbar q_{\max }=2 m v$, Eq. (2) finally leads to (with $Q=q^{2} / 2 m$ )

$$
-\left(\frac{d E}{d x}\right)_{0}=\frac{4 \pi z^{2} e^{4}}{m v^{2}} N Z \ln \left[\frac{2 m v^{2}}{I}\right),
$$

which identifies the nonrelativistic limit of Eq. (1) when $\beta^{2} \rightarrow 0$. Previously, we have obtained the corrections to Eq. (4) for a one-electron system to order $(Z \alpha)^{2}$ in the form ${ }^{12}$

$$
\Delta=-\frac{\hbar^{4}}{8 m^{3} c^{2}}\left[q^{4}+\frac{10}{3} \frac{q^{2}}{a^{2}} Z^{2}\right],
$$

where $a$ is the Bohr radius. Employing the virial theorem, this result can be rewritten in terms of the ground-state binding energy $\left(E_{\text {tot }}<0\right)$ of the atom as ${ }^{10}$

$$
\Delta=-\frac{\hbar^{4} q^{4}}{8 m^{3} c^{2}}+\frac{5}{6}\left[\frac{\hbar q}{m c}\right)^{2} E_{\text {tot }} .
$$

Incorporating the result in Eq. (7) (in the independent- 
TABLE I. Comparison of various corrections to the Bethe theory.

\begin{tabular}{ccccccc}
\hline $\begin{array}{c}\text { Target } \\
\text { element } \\
(Z)\end{array}$ & $I(\mathrm{eV})^{\mathrm{a}}$ & $\left|E_{\text {tot }}\right|(\mathrm{MeV})^{\mathrm{b}}$ & $\Delta_{R}$ & $C / Z^{\mathrm{c}}$ & $z L_{1}{ }^{\mathrm{c}}$ & $z^{2} L_{2}{ }^{\mathrm{c}}$ \\
\hline $\mathrm{Al} \mathrm{(13)}$ & 164 & $6.6 \times 10^{-3}$ & $5.4 \times 10^{-3}$ & $2.3 \times 10^{-1}$ & $1.1 \times 10^{-1}$ & $2.2 \times 10^{-2}$ \\
$\mathrm{Cu}(29)$ & 317 & $4.5 \times 10^{-2}$ & $1.3 \times 10^{-2}$ & $2.8 \times 10^{-1}$ & $8.8 \times 10^{-2}$ & $1.7 \times 10^{-2}$ \\
$\mathrm{Ag}(47)$ & 469 & $1.5 \times 10^{-1}$ & $2.2 \times 10^{-2}$ & $2.9 \times 10^{-1}$ & $1.1 \times 10^{-1}$ & $2.1 \times 10^{-2}$ \\
$\mathrm{Au} \mathrm{(79)}$ & 770 & $5.2 \times 10^{-1}$ & $3.7 \times 10^{-2}$ & $2.2 \times 10^{-1}$ & $9.8 \times 10^{-2}$ & $1.8 \times 10^{-2}$ \\
\hline \hline
\end{tabular}

${ }^{a}$ Reference 14.

${ }^{\mathrm{b}}$ Reference 16.

${ }^{\mathrm{c}}$ Experimental values from Ref. 13.

particle and local-potential limit ${ }^{10}$ ) into Eq. (4) and rearranging terms, one obtains

$$
|M|^{2}=\frac{\hbar^{2}}{2 m} Z q^{2}\left[1+\frac{5}{3} \frac{E_{\mathrm{tot}}}{Z m c^{2}}-\frac{1}{4}\left(\frac{\hbar q}{m c}\right)^{2}\right] .
$$

Substituting Eq. (8) into Eq. (2), one finally obtains the relativistic corrections to the Bethe stopping-power formula in the form

$$
\begin{aligned}
-\left[\frac{d E}{d x}\right]_{R}= & -\left[\frac{d E}{d x}\right]_{0}\left[1+\frac{5}{3} \frac{E_{\mathrm{tot}}}{Z m c^{2}}\right] \\
& -\frac{\pi N Z \hbar^{4}}{m^{2} c^{2}}\left[\frac{z e^{2}}{\hbar v}\right]^{2}\left(Q_{\max }-Q_{\min }\right) .
\end{aligned}
$$

The last term can easily be shown to be negligible for nonrelativistic incident particles plus the fact that the mean excitation energy $I \lesssim m v^{2}$ for most target atoms. Hence, our first-order correction to the Bethe formula will simply include a modification factor which can be expressed as

$$
-\left(\frac{d E}{d x}\right)_{R}=-\left(\frac{d E}{d x}\right]_{0}\left(1+\frac{5}{3} \frac{E_{\mathrm{tot}}}{Z m c^{2}}\right) .
$$

Combining with Eq. (5), Eq. (10) can be rewritten in the following form:

$$
-\left(\frac{d E}{d x}\right)_{R}=\frac{4 \pi z^{2} e^{4}}{m v^{2}} N Z L(v),
$$

where

$$
L(v)=\ln \left(\frac{2 m v^{2}}{I}\right)\left(1+\frac{5}{3} \frac{E_{\text {tot }}}{Z m c^{2}}\right) .
$$

On the other hand, it is known that there exists many other corrections to the original Bethe theory such as the shell corrections $(\sim C / Z)$, the Barkas $(\sim z)$, and the Bloch $\left(\sim z^{2}\right)$ correction terms. ${ }^{13,14}$ Incorporating all these, Eq. (12) can more generally be expressed as ${ }^{13}$
$L(v)=\ln \left(\frac{2 m v^{2}}{I}\right)-\Delta_{R}-C / Z+z L_{1}-z^{2} L_{2}$,

with the relativistic correction term defined by

$$
\Delta_{R}=\frac{5}{3} \ln \left[\frac{2 m v^{2}}{I}\right] \frac{\left|E_{\mathrm{tot}}\right|}{Z m c^{2}},
$$

with the quantities $\Delta_{R}, C, L_{1}$, and $L_{2}$ being positive. Note that if one adopts a $Z$ dependence of $\left|E_{\text {tot }}\right|$ as $\left|E_{\text {tot }}\right| \sim Z^{7 / 3}, 15$ Eq. (14) leads to $\Delta_{R} \sim Z^{4 / 3}$. Since $\left|E_{\text {tot }}\right| \ll m c^{2}$ for light elements, we expect that the effect in Eq. (14) will be significant only for heavy elements, provided that it is not outweighed by the increasing values of $I$ and $Z$ for heavy target atoms. Furthermore, Eq. (14) shows that $\Delta_{R}$ increases with the energy of the incident particles while the other corrections $\left(C / Z, z L_{1}\right.$, and $z^{2} L_{2}$ ) are expected to vanish at high incident energies, ${ }^{13,14}$ hence we expect that $\Delta_{R}$ will be most significant for very heavy target elements and with energetic incident particles. Table I shows a comparison of these correction terms for various target elements with incident protons at an energy equal to $2 \mathrm{MeV}$. It is clear that while the relativistic effect is small at this incident ener$\mathrm{gy}$, it becomes at least comparable to the Barkas and the Bloch terms for light incident particles such as proton and for heavy target elements such as gold. We have investigated this effect with the increase of incident energy for up to $50 \mathrm{MeV}$ for protons and have found that at such energies $\Delta_{R}$ becomes comparable to the shell corrections and predominates over the Barkas and Bloch terms (e.g., for $50 \mathrm{MeV}, \Delta_{R}=0.106$ and $C / Z=0.112$ for gold while both $z L_{1}$ and $z^{2} L_{2}$ are completely negligible). To conclude, we want to stress that for very high incident energies at which the shell, Barkas, and Bloch corrections are all expected to vanish, the present relativistic effect will manifest itself significantly for heavy target elements, although it might be necessary to adopt a relativistic treatment for the incident particle in such cases.

The author thanks P. Y. Feng for very useful discussions. 
${ }^{1}$ H. A. Bethe, Ann. Phys. (Leipzig) 5, 325 (1930).

${ }^{2}$ U. Fano, Ann. Rev. Nucl. Sci. 13, 1 (1963).

${ }^{3}$ Studies in the Penetration of Charged Particles, National Academy of Sciences Publication No. 1133, edited by U. Fano (National Research Council, Washington, D.C., 1964), pp. 281-284.

${ }^{4}$ J. S. Levinger, M. L. Rustgi, and K. Okamoto, Phys. Rev. 106, 1191 (1957).

${ }^{5}$ T. Matsuura and K. Yazaki, Phys. Lett. 46B, 17 (1973).

${ }^{6}$ P. T. Leung, M. L. Rustgi, and S. A. T. Long, Phys. Rev. A 33, 2827 (1986)

${ }^{7}$ M. L. Rustgi, P. T. Leung, and S. A. T. Long, Phys. Rev. A 37, 1775 (1988).

${ }^{8}$ K. M. Schmitt and H. Arenhovel, Z. Phys. A 320, 311 (1985).

${ }^{9}$ J. D. Walecka, Nucl. Phys. A399, 387 (1983); T. de Forest, Jr., Phys. Lett. 152B, 151 (1985).
${ }^{10}$ D. Y. Smith, Phys. Rev. A 35, 3381 (1987).

${ }^{11}$ See, e.g., H. A. Bethe and R. W. Jackiw, Intermediate Quantum Mechanics, 2nd ed. (Benjamin, New York, 1968), p. 304.

${ }^{12}$ Note that the extra factor $1 /(2 \gamma-1)$ obtained in the previous work due to the application of the consistent ground-state wave function in Refs. 6 and 7 is superfluous. It can be shown that this factor can only lead to corrections of $O\left((Z \alpha)^{4}\right)$, which we have been ignoring throughout our work.

${ }^{13}$ H. H. Andersen, J. F. Bak, H. Knudsen, and B. R. Nielsen, Phys. Rev. A 16, 1929 (1977).

${ }^{14}$ See also, S. P. Ahlen, Rev. Mod. Phys. 52, 121 (1980).

${ }^{15}$ See, e.g., N. H. March, Self-consistent Fields in Atoms (Pergamon, Oxford, 1975), p. 53.

${ }^{16}$ K. N. Huang, M. Aoyagi, M. H. Chen, B. Crasemann, and H. Mark, At. Data Nucl. Data Tables 18, 243 (1976). 